\title{
Naming and categorization of tilted alphanumeric characters do not require mental rotation
}

\author{
MURRAY J. WHITE \\ Victoria University of Wellington, Wellington, New Zealand
}

\begin{abstract}
Single letters and numbers were shown at different angular orientations in the frontal plane, in both forward and backward (mirror-image) versions. In three separate conditions, subjects were required to discriminate the stimuli on the basis of version, category (letter, number, and name (G, 2, etc.). There was a pronounced effect of orientation on version judgments but none at all on category and name judgments, indicating that the identification of a tilted character requires neither the assignment of a cognitive up-down axis nor mental rotation to the upright. Nevertheless, reaction times for backward versions were slower than reaction times for forward versions in both category and name conditions, implicating some sort of interhemispheric transfer process. No support was obtained for the so-called "conceptual category" effect in that reaction times for category judgments were consistently slower than reaction times for name judgments.
\end{abstract}

Our identification of objects in the real world tends toward veridicality or constancy rather than the illusory, notwithstanding considerable variations in the relative brightness, retinal image position, and proximal image size of object attributes. Likewise, fluctuations in the orientation of an object's image about the horizontal and vertical depth planes have a not too marked effect upon veridical perception. Changes in orientation with respect to the normal frontal plane can, however, make for difficult and ambiguous object identification, as is evidenced by a square tilted $45 \mathrm{deg}$ being perceived as a diamond, a " $\mathrm{d}$ " rotated $180 \mathrm{deg}$ being identified as a "p," and the difficulty people have in recognizing upside-down faces (Attneave, 1968; Rock, 1973).

A question of some importance for shape recognition theories is whether, in order to identify (recognize or name) a stimulus tilted in the frontal plane, it is first necessary to correct for tilt or "mentally rotate" the imaged object to the upright position. Corballis and his colleagues (Corballis \& Nagourney, 1978; Corballis, Zbrodoff, Shetzer, \& Butler, 1978) have recently addressed themselves to this problem in a series of experiments using the well-established Cooper and Shepard (1973) paradigm. In this paradigm, single alphanumeric characters are presented at various angular orientations with respect to the environmental upright, in both normal and backward (mirror-image) versions,

This research was supported in part by grants from the Internal Research Committee of Victoria University of Wellington and the University Grants Committee. Requests for reprints should be addressed to Murray J. White, Department of Psychology, Victoria University of Wellington, Private Bag, Wellington, New Zealand. and the subject's task is to make a rapid decision whether a character is a normal or a backward version.

Contrary to the marked effect of angular orientation upon decision reaction times (RTs) invariably observed for backward-forward judgments (e.g., Cooper \& Shepard, 1973, p. 103), Corballis et al. (1978) found RTs to be invariant across angular orientation when subjects were required to judge the name of a stimulus character (G, 2, etc.) and the conceptual category of a character (letter, number). They also found that, in both judgment tasks, RTs to backward characters were marginally but reliably slower than RTs to forward characters. The importance of these findings is twofold. First, they limit the generality of theories of shape recognition that demand some correction for environmental tilt before recognition can occur. Rock (1973), for example, has argued that "recognition is generally governed by the corrected [for tilt] percept, even with letters" (p. 50). Second, the observed backward-forward RT differences in the naming and categorization tasks are difficult to reconcile with feature-extraction theories (e.g., Milner, 1974), which predict invariance with respect to mirror-image discrimination.

The present experiment attempts to validate the findings of Corballis et al. (1978) by preserving a greater measure of internal experimental consistency. This is achieved by requiring all subjects to make all possible judgments (about version, name, and category), by not varying response probabilities, and by using a single fixed response-indicator procedure. Additionally, the logic of the design permits a reappraisal of the "conceptual category" effect (Brand, 1971; Ingling, 1972; Jonides \& Gleitman, 1972), which poses certain theo- 
retical problems for feature-extraction theories of shape recognition.

\section{METHOD}

\section{Stimuli}

Stimuli were the uppercase letters $G, J$, and $R$ and the Arabic numerals 2, 5, and 7 (Letraset Futura medium). Each of these was transferred onto plain white cards at six different orientations with respect to the upright: $0,60,120,180,240$, and $300 \mathrm{deg}$. The stimulus characters were then photographed and developed into $35-\mathrm{mm}$ positive transparencies such that, when back-projected on a screen and viewed at a distance of $1 \mathrm{~m}$, an upright stimulus subtended a vertical visual angle of 2 deg. Four copies of each of the 36 stimulus-orientation transparencies were made, and two of each of these were turned into backward (mirror-image) versions by turning the slides $180 \mathrm{deg}$ around the vertical axis before placing them in the slide tray. The total set of 144 slides was arranged in a random sequence given the constraints that no one stimulus character, no one orientation, and no one version appear more than three times in succession.

\section{Procedure}

Each stimulus trial consisted of the presentation of a cue followed by the presentation of a critical stimulus character. (1) In the version condition, a stimulus character was preceded by either the word FORWARD or the word BACKWARD. (2) In the category condition, a character was preceded by either the word LETTER or the word NUMBER. In both these conditions, the cue words were shown in capital letters in a font different from that used for the stimulus characters. (3) In the name condition, a character was preceded by one of $G, J, R$, 2,5 , and 7 , shown in a normal upright position. These cue stimuli were also shown in a font different from that used for the critical stimuli.

Each condition constituted a separate experimental session. The subject's task was to respond as rapidly as possible when, in each trial, the properties of the critical stimulus character matched the immediately preceding cue. When there was a mismatch (as there was in a random $50 \%$ of the trials in each condition), the subject was not to respond. In one trial, for example, the critical character " 7 " was shown in a backward version at an orientation of $180 \mathrm{deg}$ and was preceded in the respective conditions by the word BACKWARD, the word NUMBER, and the number 7 ; in all cases, the subject was required to respond. In all three conditions, each critical stimulus character, at each orientation and in each version, was preceded once by a match cue and once by a mismatch cue. In the name condition, mismatch cues for numbers were always letters, and for letters, always numbers. The positioning of the cues in the sequence of trials was also arranged so that not more than three response or three no-response trials followed each other in succession.

A single trial consisted of the following sequence of events: (1) A slide showing the match information was presented for $2 \mathrm{sec}$; (2) a blank interval appeared for $1 \mathrm{sec}$; (3) a stimulus character was presented for $4 \mathrm{sec}$. The interval between the onset of a stimulus character and the onset of the match cue for the next trial was $8 \mathrm{sec}$. All intervals other than those occupied by the match and character stimuli appeared blank.

Subjects were tested individually in three separate sessions, with the order of conditions counterbalanced over the six subjects. A subject sat facing a translucent screen and behind a low table on which the response key apparatus rested. The task, as explained to the subject, was to press two keys simultaneously, one with the left index finger and one with the right index finger, whenever the properties of a critical stimulus character matched the immediately preceding cue. No response was to be made in mismatch trials. A number of familiarization and practice trials were given to each subject at the beginning of each session/condition; feedback about response accuracy was provided here, but not in the actual experimental trials. Subjects were asked to put a premium on accuracy and to make as rapid a decision/response as possible. No physical attempt was made to constrain head movements, but subjects were asked to sit straight and to keep their heads in an upright position. The apparatus consisted of a three-field tachistoscopic projector with timers to control the trial sequences and to monitor response times to within $1 \mathrm{msec}$.

\section{Subjects}

Six undergraduate psychology students enrolled in a human memory course volunteered their services. None was familiar with mental rotation work at the time of testing. All were paid for participation.

\section{RESULTS}

The dependent variable of principal interest was the mean correct response latency (RT) for each subject at each orientation by version by match (judgment) condition treatment level. (Response time was the interval elapsing between onset of a critical stimulus to the first depression of a response key.) In the case of misses (1.5\% of all trials) and equipment failures (1.6\% of all trials), the missing RTs were estimated and replaced according to the procedure recommended by Winer (1971). There were $8.2 \%$ false alarms in the version match condition, $3.9 \%$ in the category match condition, and $.5 \%$ in the name match condition (i.e., there was no speed-accuracy tradeoff problem). The distributions of correct RTs are shown in Figure 1.

A four-way repeated measures ANOVA of the correct RTs (subjects by match condition by version by orientation) showed a substantial overall effect due to match condition, with version judgments being slowest (mean $=1,435 \mathrm{msec}$ ), category judgments next fastest (mean $=570 \mathrm{msec}$ ), and name judgments fastest $($ mean $=437 \mathrm{msec}) \quad[\mathrm{F}(2,10)=142.42]$. The main effects of version and orientation were also highly significant $[F(1,5)=51.29$ and $F(5,25)=34.06$, respectively]. Of greater interest, the interactions between match condition and stimulus version and between match condition and orientation were substantial $[F(2,10)=37.00$ and $F(10,50)=29.40$, respectively $]$. All these effects were statistically reliable at better than the .1 level, but neither the Version by Orientation nor the triple interaction approached an acceptable level of significance.

The nature of the two significant interactions can be readily appreciated from Figure 1. First, in the condition in which subjects were required to decide the version (backward or forward) of a character, correct backward judgments took a mean of $471 \mathrm{msec}$ longer than correct forward judgments. In the category condition, the backward-forward RT difference dropped to $24 \mathrm{msec}$, and in the name condition, it was $44 \mathrm{msec}$. Second, the effect of angular orientation was visibly limited to the version match condition.

In order to clarify these interactive effects, three separate ANOVAs were performed on the RT results for the separate conditions. The effect of backward-forward 


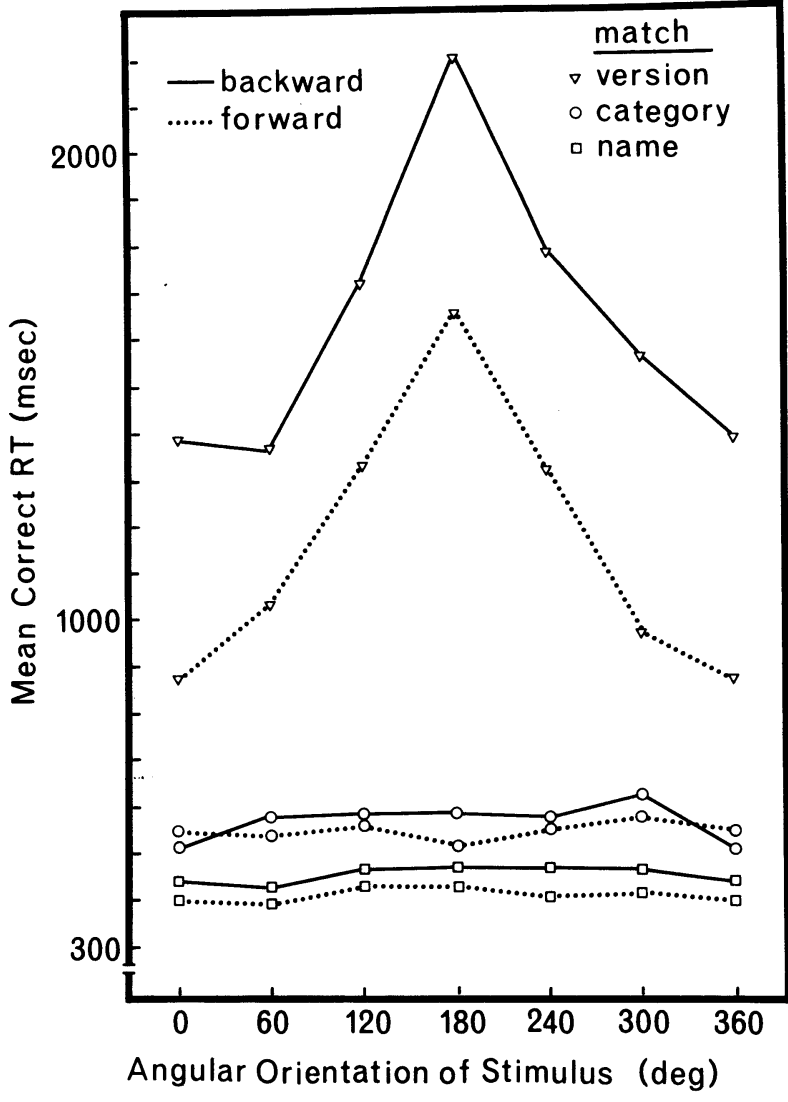

Figure 1. Mean correct decision RTs for forward and backward (mirror-image) characters at different angular orientations with respect to the upright.

version was significant in both the version condition $[F(1,5)=43.42]$ and in the name condition $[F(1,5)=$ $16.24]$. In the category condition, this effect only approached reliability $[\mathrm{F}(1,5)=5.30, .05<\mathrm{p}<.10]$, due to a crossover for versions observed for 0 -deg (upright) characters (see Figure 1). Only in the version condition was the effect of angular orientation significant $[F(5,25)=38.89]$, and in none of these three ANOVAs was any interaction found between version and angular orientation.

\section{DISCUSSION}

The results of this experiment are in essential agreement with those reported by Corballis et al. (1978). One slight difference, probably attributable to differences in experimental procedures, is that the RT functions presently observed for category and name conditions are much more invariant with respect to angular orientation than are those shown by Corballis et al. In addition, the present experiment has generated internally consistent RT data for the critical version condition.

Inspection of Figure 1 suggests a common process underpins the naming and the categorizing of rotated characters, while some additional (or separate) process is required to decide the version of a tilted character. The results are consistent with the following descriptive processes. In both name and category conditions, the cue information is used to establish an internal representation of a set of stimulus features against which the critical stimulus image is then tested. These features or featural relationships are invariant with respect to angular orientation; the fewer the test features and the less ambiguous the featural relationships, the more accurate and rapid the test match will be. Thus, for example, the cue " $R$ " might establish a test based on the featural relationship between a straight line and a closed loop that is sufficient, whatever the orientation or version of the critical stimulus, to determine the correct name of the stimulus "R." Exactly the same process might be responsible for decisions about the category of a character, except, of course, there will now be an increase in the number of feature tests that need to be made. In neither the naming nor the category conditions does the subject need to adjust the orientation of the stimulus image. When deciding the version of the character, however, such adjustment is necessary, and the internal representation of the discriminated character must be mentally rotated to the upright for a decision about its version (Cooper \& Shepard, 1973).

This description appears to be sufficient to account for the most salient results, namely: (1) the absence of angular orientation effects in the name and category conditions, (2) the observation that decisions about category required (on average) $133 \mathrm{msec}$ longer than decisions about name, and (3) the observed interaction between condition and stimulus version. As far as the second of these results is concerned, the results confirm the previous findings of White (1977) in showing that the categorization of alphanumeric stimuli does not preempt their naming and/or identification. The "conceptual category" effect (wherein, for example, a digit is identified more rapidly in the context of letters than it is in the context of other digits) can be satisfactorily accommodated in terms of between-category stimulus featural differences and such gross differences as mean stimulus width.

With respect to the Condition by Version interaction, it is clear that, whereas naming and categorization do not require mental rotation, the version of a stimulus does have some effect on the decision process. The magnitude and consistency of these RT differences (cf. Corballis et al., 1978) implicate interhemispheric transfer processes as the probable locus (Geffen, Bradshaw, \& Nettleton, 1972). Indeed, Cohen (1975) has found that for version judgments, rotated alphanumeric characters are discriminated faster in the left visual field than in the right visual field. She has concluded that "de-rotation is always carried out in the right hemisphere, stimuli being transmitted across the corpus callosum when presented initially to the left hemisphere" (Cohen, 1975, p. 29). However, Cohen's experiment was not primarily aimed at answering the questions of present concern (neither naming nor category decisions were required, and separate backward and forward RTs were not reported). In order to nail down the locus of these backward-forward RT differences, a further experiment is needed in which visual field stimulus position is incorporated as a factor.

Finally, it must be said that the above analysis is of necessity restricted to version, category, and name decisions about overlearned stimuli that are readily featurally discriminable. Still, the present findings, together with those of Corballis et al. (1978) would seem to limit the generality of theories that maintain that object (letter) recognition is made of the tiltcorrected percept (e.g., Rock, 1973) and of those that predict invariant perceptual decisions for mirror-image and rotated stimulus images (e.g., Milner, 1974).

\section{REFERENCES}

Atrneave, F. Triangles as ambiguous figures. American Journal of Psychology, 1968, 81, 447-453.

Brand, J. Classification without identification in visual search. Quarterly Journal of Experimental Psychology, 1971, 23, 178-186.

Conen, G. Hemispheric differences in the utilization of advance information. In P. M. A. Rabbit \& S. Dornic (Eds.), Attention and performance, Vol. 5. London: Academic Press, 1975.

Cooper, L. A., \& ShePARD, R. N. Chronometric studies of the 
rotation of mental images. In W. G. Chase (Ed.), Visual information processing. New York: Academic Press, 1973.

Corballis, M. C., \& Nagourney, B. A. Latency to categorize disoriented alphanumeric characters as letters or digits. Canadian Journal of Psychology, 1978, 23, 186-188.

Corballis, M. C., Zbrodoff, N. J., Shetzer, L. I., \& Butler, P. B. Decisions about identity and orientation of rotated letters and digits. Memory \& Cognition, 1978, 6, 98-107.

Geffen, G., Bradshaw, J. L., \& Nettleton, N. C. Hemispheric asymmetry: Verbal and spatial encoding of visual stimuli. Journal of Experimental Psychology, 1972, 95, 25-31.

INGLiNG, N. W. Categorization: A mechanism for rapid information. Journal of Experimental Psychology, 1972, 94, 239-243.
Jonides, J., \& Gleitman, H. A conceptual category effect in visual search: $\mathrm{O}$ as letter or as digit. Perception \& Psychophysics, 1972, 12, 457-460.

Milne R, P. M. A model for visual shape recognition. Psychological Review, 1974, 81, 521-535.

Rock, I. Orientation and form. New York: Academic Press, 1973. WhITE, M. J. Identification and categorization in visual search. Memory \& Cognition, 1977, 5, 648-657.

WINER, B. J. Statistical principles in experimental design (2nd ed.). New York: McGraw-Hill, 1971.

(Received for publication October 22, 1979.) 